\title{
Learning support and academic achievement among Malaysian adolescents: the mediating role of student engagement
}

\begin{abstract}
The aim of this study was to examine the associations between learning support, student engagement and academic achievement among adolescents. We also examined the extent to which affective, behavioural and cognitive engagement play a mediating role in studentsô perceived learning support from parents, teachers and peers, and contribute to their academic achievement. Malaysian adolescents (aged $12 i ̈ 17$ years, $\mathrm{N}=2359$ ) completed a selfadministered questionnaire based on an adapted version of the Student Engagement And Learning Support Scale. Item and factor analyses were performed to ensure appropriate psychometric properties of the scales. Pearson correlation analysis identified the relationship between variables and structural equation modelling was conducted to identify the role of student engagement as a mediator between learning support and academic achievement. The study provides empirical support for the hypothesis that perceptions of learning support influence adolescentsôaffective, behavioural and cognitive engagement in school in different ways, which in turn influences their academic achievement. Cognitive engagement seemed to be the best predictor of academic achievement and the strongest mediator for all three types of learning support. Behavioural engagement was negatively associated with academic achievement, and affective engagement did not have a direct relationship with academic achievement, although it contributed indirectly through cognitive and behavioural engagement. The results of this study provide a basis for policy makers to initiate prevention and intervention programs for increasing the quality of parentï child, teacherï student and peerï peer relationships which ultimately could lead to improved academic competence and outcomes.
\end{abstract}

Keyword: Affective engagement; Behavioural engagement; Cognitive engagement; Parental support; Peer support; Teacher support 